\title{
DUST, STAR, AND HYDROGEN DISTRIBUTIONS IN THE ORION ASSOCIATION
}

\author{
SYUZO ISOBE \\ Tokyo Astronomical Observatory, University of Tokyo, Mitaka, Tokyo
}

\begin{abstract}
From the star counts on the Palomar Sky Survey prints, dust and star distributions in the Orion Association are studied. The Hydrogen distribution in the same region is obtained from an $\mathrm{H} \alpha$ photograph. It is found that a considerable part of the interstellar absorption on the line of sight is due to the circumstellar dust grains in the Orion Association.
\end{abstract}

\section{Introduction}

The age of the Orion Association is estimated to be $3 \times 10^{6} \mathrm{yr}$ (Walker, 1969; Menon, 1958; Blaauw and Morgan, 1954). In this Association is the Orion Nebula whose age is said to be $10^{4}-10^{6}$ yr (Parenago, 1953; Vandervoort, 1963; Sharpless, 1966). As was already shown by the present author (Isobe, 1971), in $3 \times 10^{6}$ yr a considerable part of the gas and dust surrounding early type stars should be blown out to the surrounding space. The Orion Association is relatively near to the Sun (Johnson and Hiltner, 1956) in the univrese and high enough in galactic latitude to study the apparent distributions of dust, gas, and stars without serious disturbances by the fore-ground stars and interstellar matter.

Menon (1958) obtained the distribution of neutral hydrogen in the Orion Association by $21 \mathrm{~cm}$ observations, and found that the neutral hydrogen atoms distribute with a shape of shell. O'Dell et al. (1967) and Henize et al. (1967) detected the near ultraviolet radiation in the Orion Association, which was identified as the scattered radiation by dust grains. However, Henry and Carruthers (1970) did not detect far ultraviolet radiation within the accuracy of their observations. These situations may show the fact that the scattering coefficients of dust grains at the far ultra-violet wavelengths are small (Nandy and Wickramasinghe, 1971). Photometric and spectroscopic observations of stars in the Orion Association have been made by many observers (e.g., Sharpless, 1952; Parenago, 1954; Straizus, 1963, 1964; Bartkus, 1964). Especially, Parenago (1954) presented the distributions of stars classified by spectral type and luminosity.

In this paper, we shall first study the distributions of dusts and stars from the star counts on the Palomar Sky Survey prints and the distribution of hydrogen atoms from an $\mathrm{H} \alpha$ photograph taken by the present author, and secondly compare these results with the previous results.

\section{2. $\mathrm{H} \propto$ Observations}

An $\mathrm{H} \alpha$ photograph was taken by the Nikon $\mathrm{F}$ camera attached to the head of the $12^{\prime \prime}$ telescope at the Okayama Astrophysical Observatory. The camera has an aperture of 
$5 \mathrm{~cm}$ with a focal length $5 \mathrm{~cm}$, which corresponds to a film scale of $70^{\prime}$ per milimeter. Figure 1 shows a reproduction of the photograph.

From the photograph, isodensity contour maps were obtained with the isodensito-

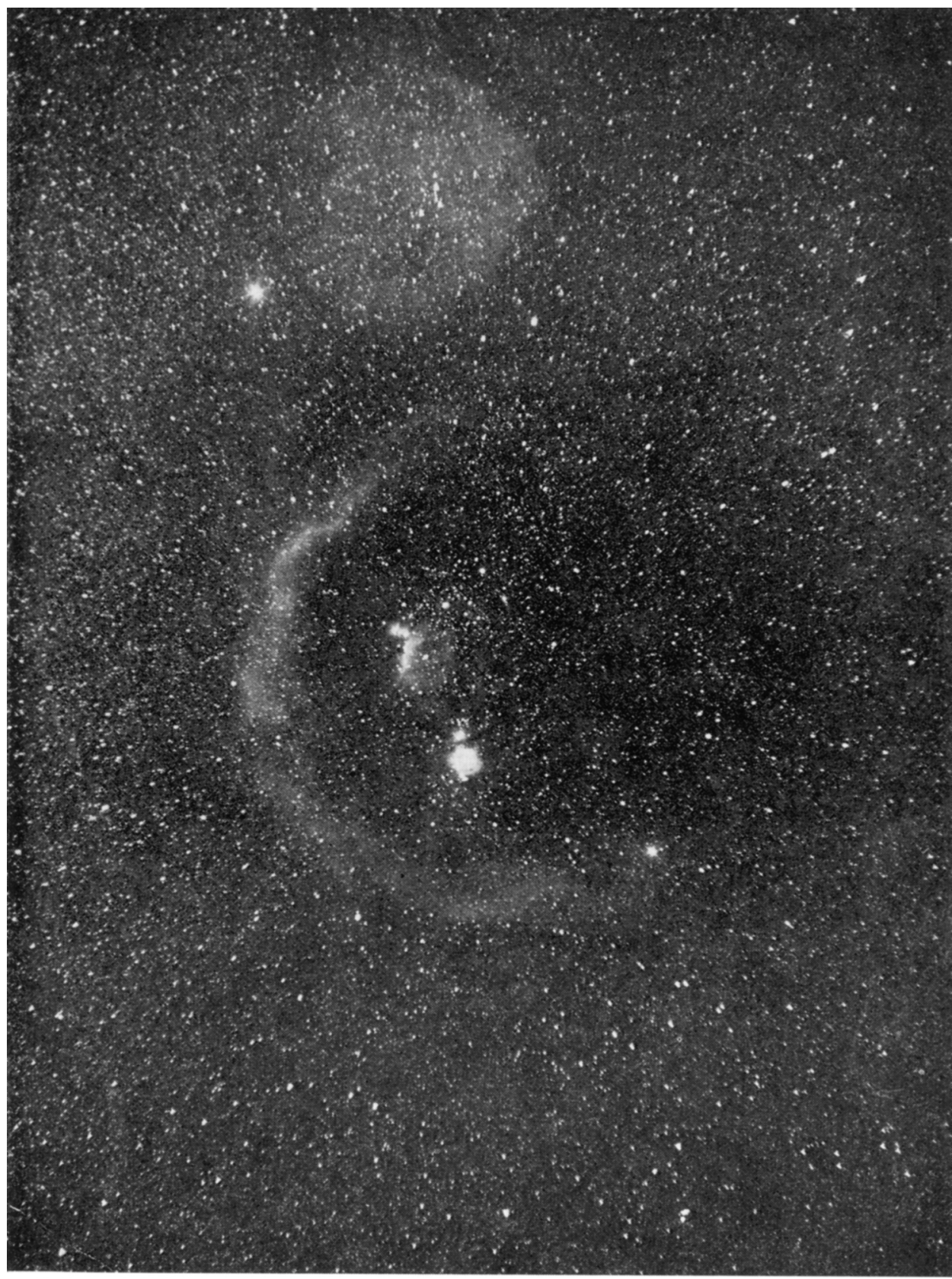

Fig. 1. $\mathrm{H} \alpha$ photograph of the Orion Association. Up is north and left is east. 
TABLE I

The data of $\mathrm{H} \alpha$ observations

\begin{tabular}{ll}
\hline Date & 1969 January 17 \\
\hline Exposure time & 100 minutes \\
Emulsion & Kodak 103a-E \\
Filter & Hoya R-64 \\
Central wavelength & $6563 \mathrm{~A}$ \\
Effective band width & $297 \mathrm{~A}$ \\
\hline
\end{tabular}

b

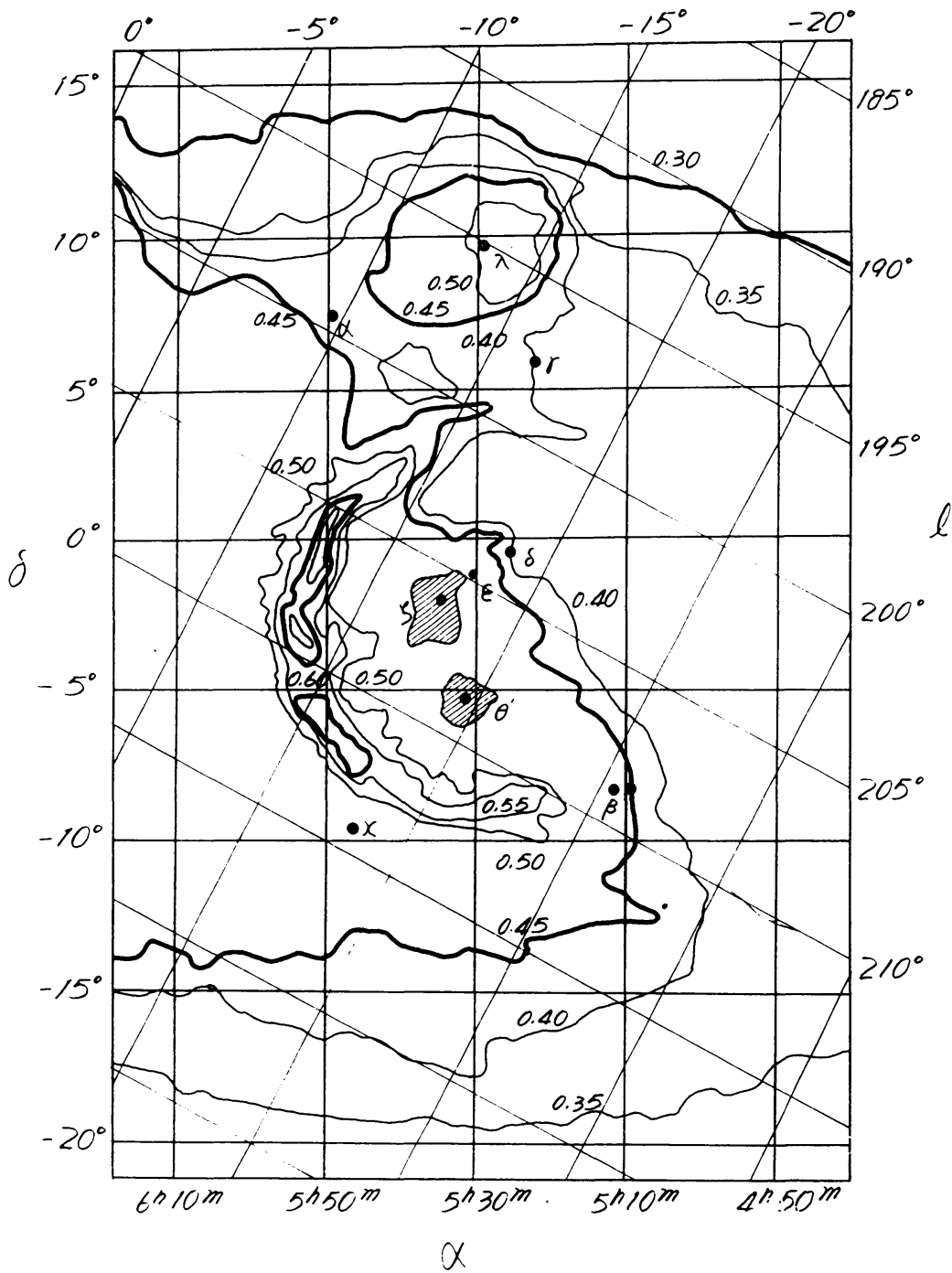

Fig. 2. Contour maps of $\mathrm{H} \alpha$ radiation in the Orion Association. The contour units are shown by plate density. The scale of all figures in this paper are same. The positions of the bright stars are shown. 
TABLE II

The relations between plate darkness and $\log$ (intensity + constant)

\begin{tabular}{ll}
\hline Plate density & $\log$ (intensity+constant) \\
\hline 0.40 & 0.84 \\
0.45 & 1.11 \\
0.50 & 1.19 \\
0.55 & 1.32 \\
0.60 & 1.39 \\
0.65 & 1.47 \\
\hline
\end{tabular}

meter of Tokyo Astronomical Observatory. In making the maps, the procedure was the same as that used by Ishida and Ohashi (1967). Date, exposure time, emulsion of film, filter, central wavelength, and effective band width are given in Table I. Figure 2 shows the resulting brightness contour map. The relations between plate darkness and logarithm of intensities plus constant are shown in Table II. This contour map indicates that the Barnard Loop in $\mathrm{H} \alpha$ radiation constitutes part of a elliptical ring. In this figure, the nebula of the Orion Association II around $\lambda$ Ori is also found.

\section{Star Counts}

Numbers of stars were counted on the blue and red prints of the Palomar Sky Survey. Each print was divided into $26^{\prime} \times 26^{\prime}$ regions. After the numbers of stars were counted for the whole region on each print, numbers of stars for several diagonal regions were counted again. The difference between counted numbers of stars obtained from two different counts was found to be less than $5 \%$. In Figures $3 \mathrm{a}$ and $3 \mathrm{~b}$ are shown the contour maps of numbers of stars on the blue and red prints.

It is found that the stars are distributed in the shape of an elliptical ring except in the region south-east from $\theta^{1}$ Ori. These distributions are similar to those of $\mathrm{H} \alpha$ radiation shown in Figure 2 and of $21 \mathrm{~cm}$ and near ultra-violet radiations as shown in a later Section. The Orion Association is at rather high galactic latitude, and therefore, the background stars might be so few to have no effects on our stars counts. Since the fore-ground of the Orion Association does not contain any clusters and nebulae and the minimum numbers of stars per $26^{\prime} \times 26^{\prime}$ region on our Figures $3 \mathrm{a}$ and $3 \mathrm{~b}$ is less than 100 , the fore-ground stars have little effect on our star counts. In these circumstances, it would be reasonable to assume that most of the faint stars observed in the direction of the Orion Association belong to this Association.

The limiting magnitudes of the blue and red original plates in Selected Area are 21.1 and 20.0 mag. respectively (Minkowski and Abell, 1963), but the corresponding ones for the blue and red prints are 20.0 and 17.5 mag., respectively, as deduced for the regions of the north equatorial pole and the north ecliptic pole (Tanabe and Mori, 1971). Since the 'Catalogue of Plates of the Palomar Sky Survey' shows that the observing conditions were similar for all plates and since the reproductions of the 


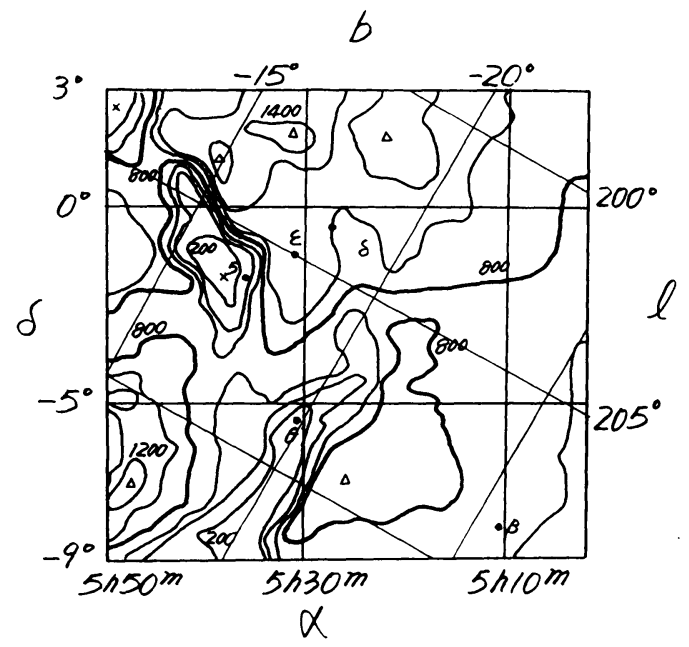

(a)

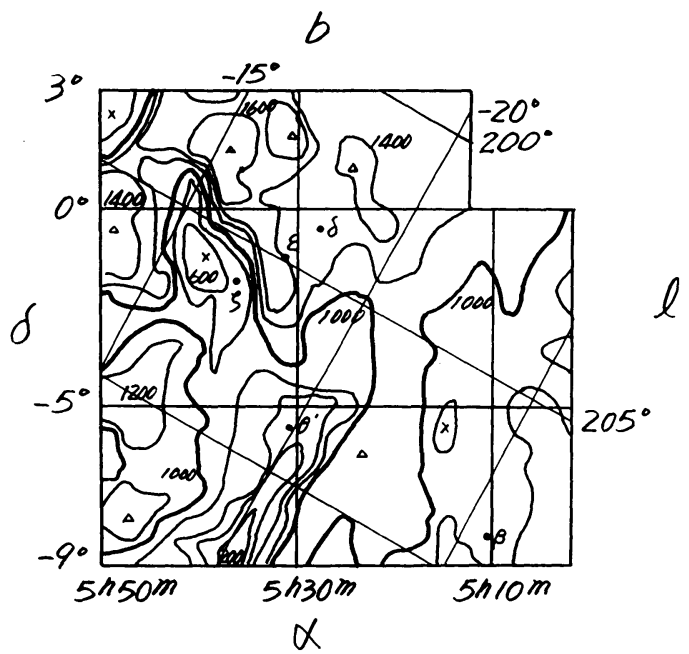

(b)

Fig. 3. (a) Contour maps of the number densities of stars in the Orion Association on the blue prints of Palomar Sky Survey. The numbers of stars in regions of $26^{\prime} \times 26^{\prime}$ are shown. (b) Same as (a), but on the red prints.

paper prints were performed with care (Minkowski and Abell, 1963) limiting magnitudes of the blue and red prints in the region of Orion Association may be taken to be 20.0 and 17.5 mag. with little risk.

The distance modulus, $m_{0}-M$, of the Orion Association is known to be 8.1 
(Johnson and Hiltner, 1956; Sharpless, 1952) and can be written as

$$
m_{0}-M=m-M-A,
$$

where $m$ is the apparent magnitude, $M$ the absolute magnitude and $A$ the magnitude of interstellar absorption. It is shown in the next section that the interstellar absorption at the visual wavelength is less than $0.6 \mathrm{mag}$. for the faint stars in the Orion Association, and the mean interstellar absorption at the same wavelength is about 0.5 mag. for the O-, B-, A-, and F-type stars in the Orion Association (Sharpless, 1952). Therefore, the interstellar absorptions at the blue and red wavelengths are of the order of $0.6 \mathrm{mag}$. and $0.3 \mathrm{mag}$, respectively.

The absolute magnitudes of stars with the limiting magnitudes of 20.0 and 17.5 mag. on the blue and red prints are 11.3 and 9.1 magnitudes, respectively. Since the Orion Association is very young, the stars with these absolute magnitudes should be dwarf or pre-main sequence stars and have temperatures of around $3000 \mathrm{~K}$. The mean color, $(B-V)$, and the bolometric correction of the $\mathrm{M}$ dwarf stars with temperatures of about $3000 \mathrm{~K}$ are not available at present, but those of stars with luminosity class I and III were obtained by Johnson (1964). From the comparisons of Johnson's (1964) Tables 3, 4, and 5 which give the mean colors and the bolometric corrections of stars of luminosity class III, I, and V respectively, the mean color of K-type stars with luminosity class III is only 0.1 mag. different with that of class $\mathrm{V}$ while the bolometric correction of the same stars is $0.5 \mathrm{mag}$. different. Therefore, the value of $1.7 \mathrm{mag}$. may be taken for the mean color of M5 dwarf stars. As is shown by Johnson (1964), the relation between $(B-V)$ and $(P-V)$ is given by

$$
(B-V)=1.19(P-V)-0.10,
$$

for the color, $B-V$, given greater than $1.0 \mathrm{mag}$. The relation between $(P-V)$ and $(P-R)$ is also given by Minkowski and Abell (1963) to be

$$
(P-R)=1.6(P-V),
$$

where $P, B, V$, and $R$ are the photographic, the blue, the visual, and the red magnitudes for a star, respectively. For the $(B-V)$ of $1.7 \mathrm{mag}$., $(P-V)$ and $(P-R)$ should be 1.5 and 2.4 mag. respectively. Therefore, the absolute magnitude at the visual wavelength is deduced to be 9.8 or 10.0 mag. from the limiting magnitudes of the blue or red prints, respectively.

Since the bolometric corrections of M-type stars were determined only from the observations of $I, J, K$, and $L$ magnitudes by Johnson (1964), they may have errors of one magnitude or so because the intensity of molecular absorption band and the effect of blanketing are different from star to star. However, at the present stage we have no data available for these problems, and therefore we adopt-2.5-3.5 mag. for the bolometric correction of the star with temperatures of about $3000 \mathrm{~K}$ according to Johnson's (1964) Table 3. Therefore, the bolometric magnitudes of the faintest stars on the blue and red prints are to be 6.5-7.5 mag.

Hayashi (1961) and Ezer and Cameron (1967) calculated the paths of stellar evolu- 
tions on the HR diagram in the early phases of the gravitational contraction and obtained the curves of the constant age for stars on the HR diagram. According to their calculations, the stars with temperature of $3000 \mathrm{~K}$ should have the bolometric magnitude of $6.5 \mathrm{mag}$. at the age of $3 \times 10^{6} \mathrm{yr}$ and situate at the pre-main sequence stage. These calculations are well consistent with our observational results.

It is concluded in this section that the stars with the limiting magnitude of $20.0 \mathrm{mag}$. on the blue prints are the same ones with the limiting magnitude of $17.5 \mathrm{mag}$. on the red prints if there is no interrtellar absorption in the region of the Orion Association.

\section{Interstellar Extinction in the Direction of the Orion Association}

We assume that the luminosity function is the same all over the Orion Association. In the absence of interstellar absorption, the apparent number of stars with a certain intrinsic luminosity in a unit area on the plane of sky is given by

$$
N_{0}=\alpha r_{0}^{3}
$$

while the apparent luminosity is

$$
L_{0}=\beta r_{0}^{2}
$$

where $\alpha$ and $\beta$ are constants depending on the space density of stars and the intrinsic luminosity, respectively, and $r_{0}$ is the distance of the center of the Association. We denote the quantities associated with the red prints and the blue prints by the subscripts $R$ and $P$ respectively, and then we obtain

$$
N_{R_{0}} / N_{P_{0}}=\left(L_{R_{0}} / L_{P_{0}}\right)^{\frac{3}{2}}
$$

leading to

$$
m_{P_{0}}-m_{R_{0}}=\frac{5}{3} \log \left(N_{R_{0}} / N_{P_{0}}\right),
$$

where $m_{R o}$ and $m_{P o}$ are the respective apparent magnitudes in the case without interstellar absorption. In the presence of interstellar absorption on the line of sight, we denote each quantity shown in Equations (4), (5), (6), and (7) by omitting the subscript 0 . In doing so, we have

$$
m_{P}-m_{R}=\frac{5}{3} \log \left(N_{R} / N_{P}\right) \text {. }
$$

By taking the difference between expressions (7) and (8), it follows that

$$
\begin{aligned}
E_{P-R} & =\left(m_{P}-m_{R}\right)-\left(m_{P_{0}}-m_{R_{0}}\right) \\
& =\frac{5}{3}\left[\log \left(N_{R} / N_{P}\right)-\log \left(N_{R_{0}} / N_{P_{0}}\right)\right] .
\end{aligned}
$$

From the results of Section 3, the stars observed on the blue prints should be the same as the stars observed on the red prints if the amounts of interstellar reddening are small. Therefore, we have adopted $N_{R 0} / N_{P o}=1.0$.

The amounts of reddening for the wavelength range from $4000 \AA$ to $7000 \AA$ are nearly inversely proportional to the wavelength in the normal reddening region and 


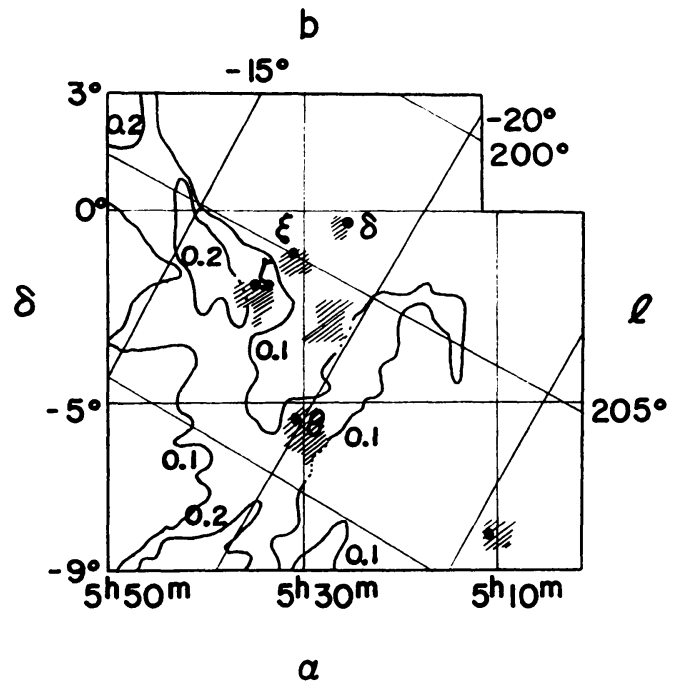

Fig. 4. Contour maps of the color excess, $E_{B-V}$, obtained from the star counts. The contour units are shown by magnitude. The numbers of stars are not counted in the hatched region.

even in the abnormal reddening region. Therefore, we shall take the wavelengths $4200 \AA, 4500 \AA, 5500 \AA$, and $6500 \AA$ for the effective wavelengths of $P, B, V$, and $R$ respectively. Thus we have

$$
E_{B-V}=E_{P-R} / 2.0 \text {. }
$$

In Figure 4 are shown the contour maps of the color excess, $E_{B-V}$.

Figure 5 shows the mean interstellar absorption of stars for each region as quoted from Figure 1 of an earlier paper (Isobe, 1971). The region extending from north to south in the parts east of $\zeta$ Ori has in some parts a color excess, in $E_{B-V}$, larger than $0.3 \mathrm{mag}$. in Figures 4 and 5 . However, the regions with a color excess, $E_{B-V}$, larger than $0.3 \mathrm{mag}$. in Figure 5 except the above region dont have such a large color excess, $E_{B-V}$, in Figure 4. Reddish (1967) showed that the amount of dust grains surrounding stars depends on the spectral types of stars and that there are few dust grains surrounding stars with spectral types later than G-type. Isobe (1971) showed that a considerable part of interstellar absorption for the O-, B-, and A-type stars in the direction of the Orion Association is caused by circumstellar grains. The color excess shown in Figure 4 is mainly due to the late type stars such as K- and M-type, while the color excess in Figure 5 is due to the early type stars such as O-, B-, A-, and F-type. Therefore, it may be concluded that the most part of interstellar absorption is caused by the circumstellar dust grains in the Orion Association except for the eastern part of $\zeta$ Ori.

\section{Comparison of Contour Maps}

The scale of the contour maps is the same. In Figure 6 is shown the contour map of the intensity of $21 \mathrm{~cm}$ radiation which is obtained from Figure 8 in van Woerden's work 


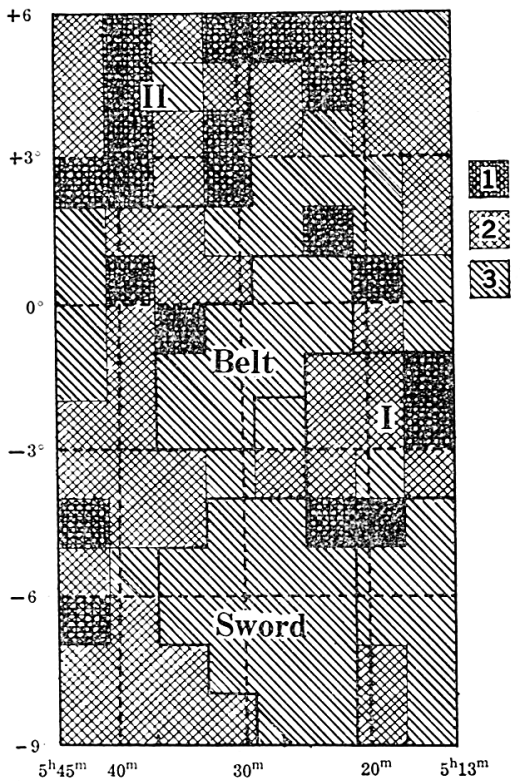

Fig. 5. Distribution of interstellar absorption obtained by Isobe (1971) from the observations of O-, B-, A-, and F-type stars. Hatched regions 1 show a mean color excess greater than 0.3 mag.; regions 2 show a mean color excess greater than 0.10 mag.; regions 3 show a mean color excess smaller than 0.10 mag.

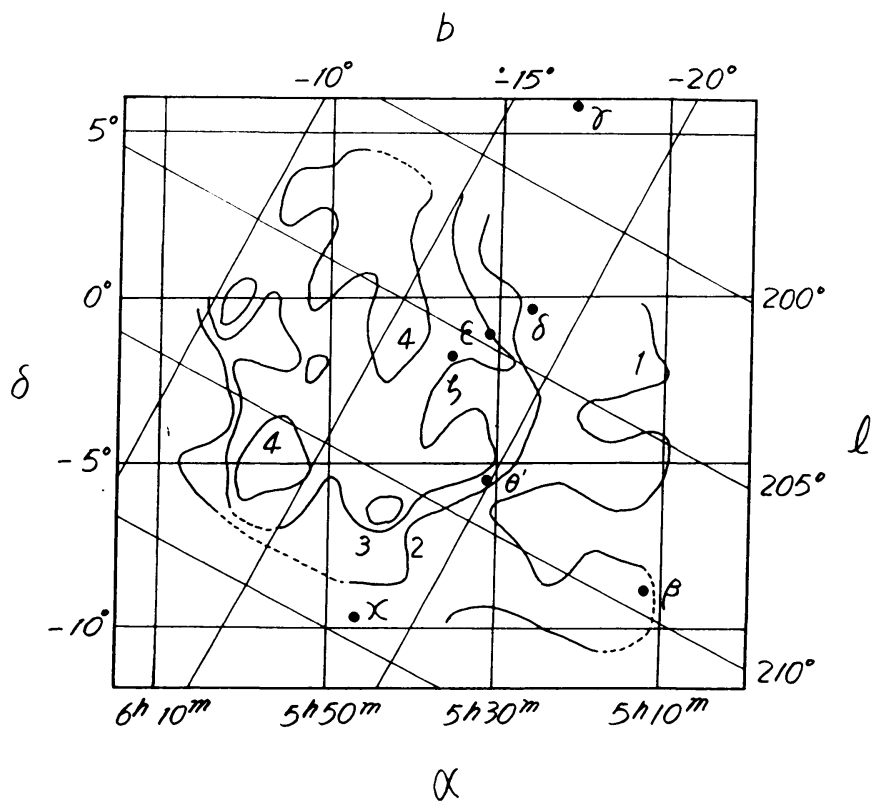

Fig. 6. Intensity distribution of the $21 \mathrm{~cm}$ radiation obtained from van Woerden's Figure 8 . Large numbers of the contour unit correspond to larger values of the equivalent width. 


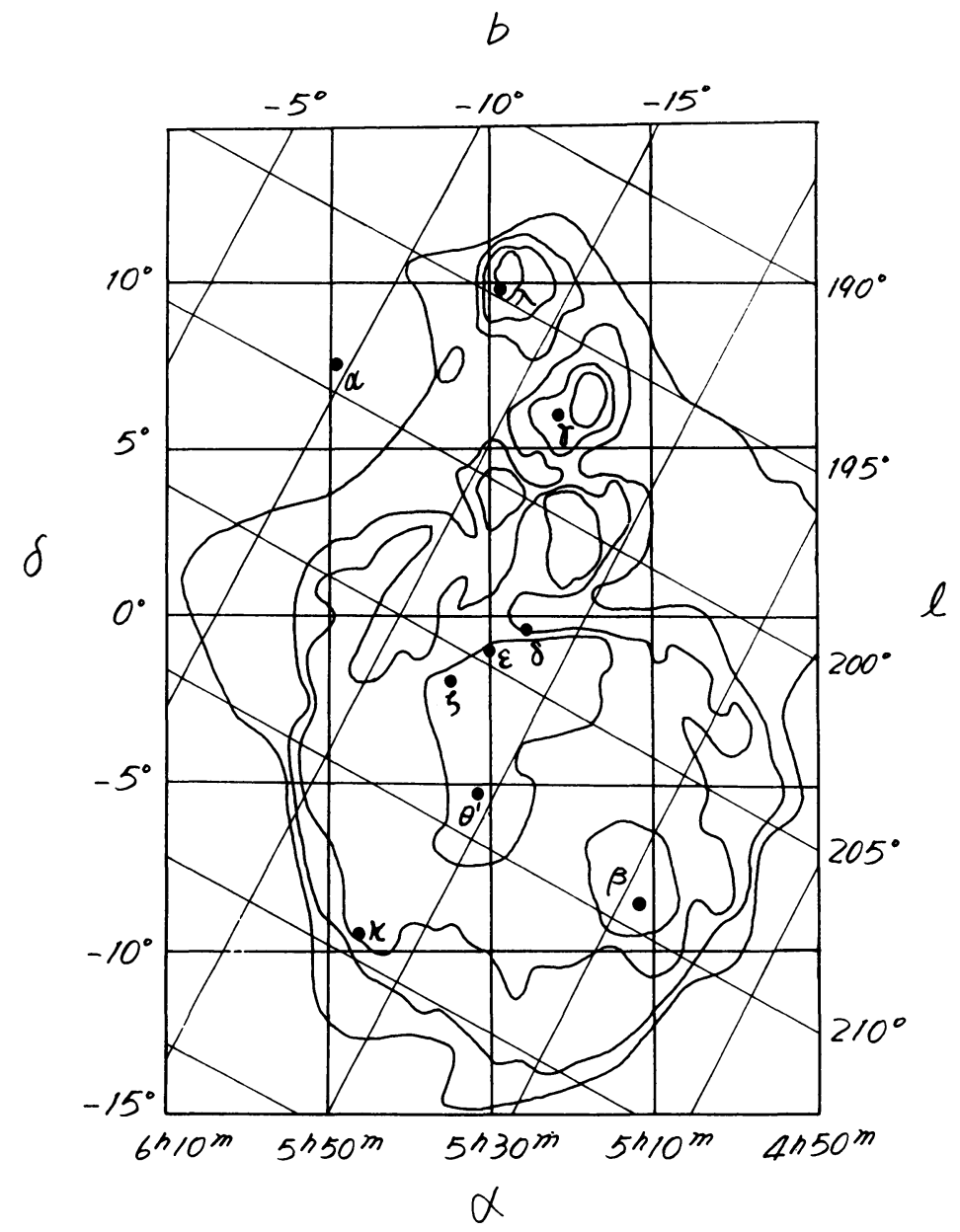

Fig. 7. The intensity distribution of the $2200 \AA-4900 \AA$ radiation obtained from the O'Dell et al.'s (1967) Figure 4. The countour units are the origional ones and are in arbitrary intensity units.

(1967). In Figure 7 is also shown the contour map of the intensity of $2200 \AA-4900 \AA$ radiation which is obtained from O'Dell et al.'s (1967) Figure 4 and which is considered to be the scattered light by dust grain. However, the contour map shown in Figure 7 does not directly show the distribution of dust grains because the observed intensity depends not only the amount of dust grains but also on the brightness of the illuminating stars and the distance of dust grains from the stars.

In all figures shown in this paper, we can easily recognize ring structures. Each ring has a marked ellipticity and the major axis of ellipse is approximately parallel to the galactic equator. It was suggested by Menon (1958) that the galactic magnetic field is the possible mechanism which can produce a distortion of an initially spherical cloud situated in the Galaxy. In Figures 3a and 3b, it is easily seen that the star density is small in the region from $\theta^{1}$ Ori to the south-east direction. This feature is quite unique. 


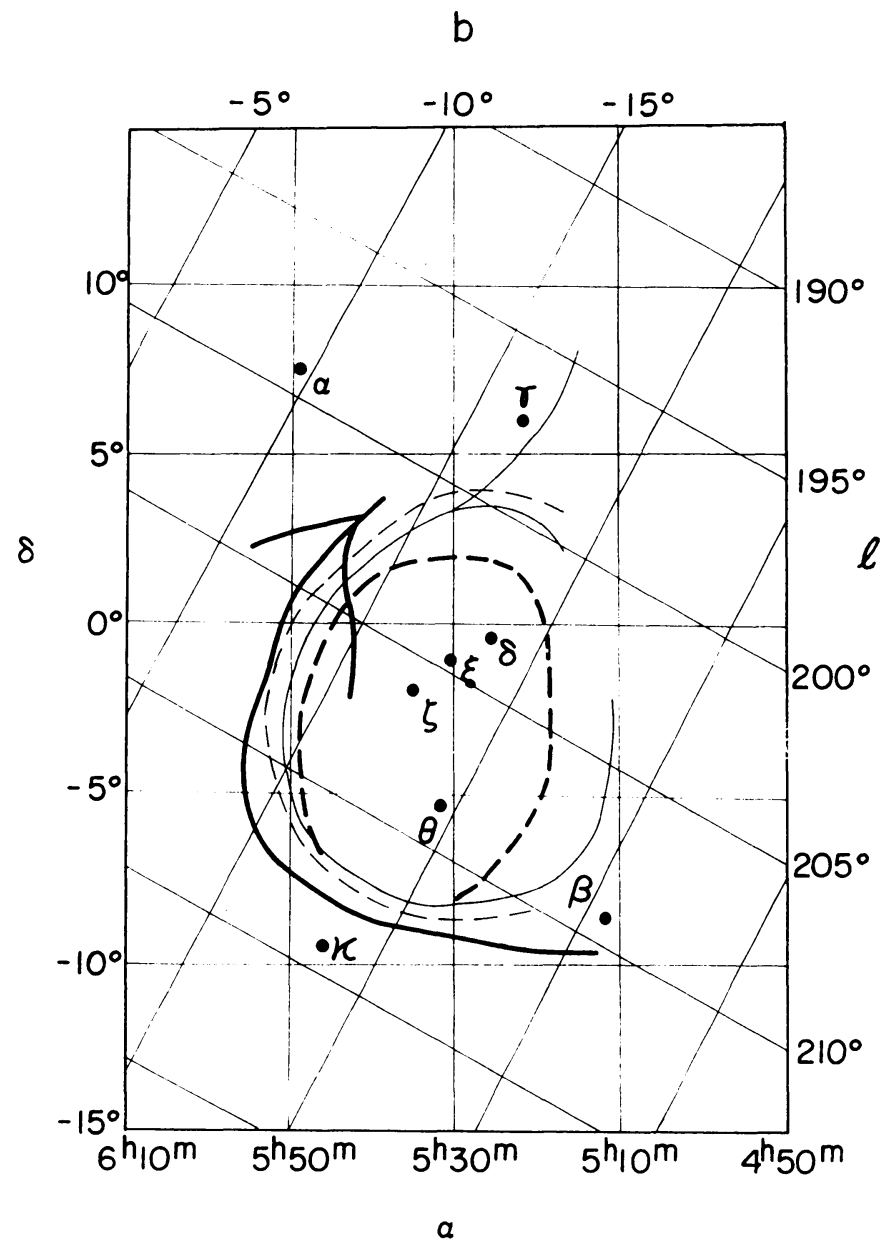

Fig. 8. Comparisons of the contour maps shown in this paper. Thick solid line shows the ridge positions of the $21 \mathrm{~cm}$ intensity; thin solid line shows the ridge positions of $2200 \AA-4900 \AA$ radiation; thick broken line shows the ridge positions of the star density; thin broken line shows the ridge positions of the $\mathrm{H} \alpha$ radiation.

As this region is also parallel to the galactic equator and would be parallel to the initial galactic magnetic line, the interaction of ionized gas and galactic magnetic field would be weak in the direction, and therefore the formation of ionized gas condensation is hard.

Figure 8 permits a comparison of the ridge positions of the contour maps. Those referring to the neutral hydrogen atoms $(21 \mathrm{~cm})$ are found to extend farthest east. According to Menon (1958), those atoms are also expanding with a velocity $10 \mathrm{~km}^{-1}$. Figure 8 also reveals that the ionized hydrogen atoms $(\mathrm{H} \alpha)$ make a fine loop in the inner part of the neutral hydrogen region. $3 \times 10^{6} \mathrm{yr}$ ago, a big explosion might have happened in the Orion Association. This explosion energy is considered to be trans- 
ferred to the $\mathrm{H} \alpha$ radiation through collisional excitation. The ridge of number density of stars is located in the inner part of the ionized hydrogen region. In the western part, the amounts of stars and dust grains are as much as in the eastern part, although the amount of hydrogen atoms is small. It is considered that the hydrogen gas expanding toward the galactic plane piles up the hydrogen gas which originally existed in interstellar space, but that the expanding hydrogen gases receding from the galactic plane did not pile up the hydrogen gases because in this region the hydrogen gas density is low before explosion. This implies that the stars are formed in the stage that the velocity of expanding gases is lower than $10 \mathrm{~km} \mathrm{~s}^{-1}$.

\section{Acknowledgements}

The author would like to express his sincere thanks to Dr K. Ishida for his helpful advice and constant encouragement, to Prof. M. Kitamura and Prof. B. Takase for their helpful discussions, and to Mr Y. Norimoto for his kindness in taking $\mathrm{H} \alpha$ photograph.

\section{References}

Bartkus, R.: 1964, Astron. Obs. Biul. Vilnius 13, 29.

Blaauw, A. and Morgan, W. W.: 1954, Astrophys. J. 119, 625.

Ezer, D. and Cameron, A. G. W.: 1967, Can. J. Phys. 45, 3461.

Hayashi, C.: 1961, Publ. Astron. Soc. Japan 13, 450.

Henize, K. G., Wackerling, L. R., and O'Callagham, F. G.: 1967, Science 155, 1407.

Henry, R. C. and Carruthers, G. R.: 1970, Science 170, 527.

Ishida, K. and Ohashi, M.: 1967, Tokyo Astron. Bull., 2nd Ser., No. 178.

Isobe, S.: 1971, Publ. Astron. Soc. Japan 23, 371.

Johnson, H. L.: 1964, Boletin de los Observatorios Tonantzintla y Tacubaya 3, 305.

Johnson, H. L. and Hiltner, W. A.: 1956, Astrophys. J. 123, 267.

Menon, T. K.: 1958, Astrophys. J. 128, 28.

Minkowski, R. L. and Abell, G. O.: 1963, Stars and Stellar Systems 3, 471.

Nandy, K. and Wickramasinghe, N. C.: 1971, Monthly Notices Roy. Astron. Soc. 154, 255.

O'Dell, C. R., York, D. G., and Henize, K. G.: 1967, Astrophys. J. 150, 835.

Parenago, P. P.: 1953, Astron. Zh. 30, 249.

Parenago, P. P.: 1954, Trudy. Gas. Astron. Inst. Sternberga 25, 1.

Reddish, V. C.: 1967, Monthly Notices Roy Astron, Soc. 135, 251.

Sharpless, S.: 1952, Astrophys. J. 116, 251.

Sharpless, S.: 1966, Vistas in Astronomy 8, 127.

Straizus, B.: 1963, Astron. Obs. Biul. Vilnius 7, 1.

Straizus, B.: 1964, Astron. Obs. Biul. Vilnius 9, 4.

Tanabe, H. and Mori, K.: 1971, Publ. Roy. Obs. Edinburgh 8, 173.

Vandervoort, P.: 1963, Astrophys. J. 138, 294.

Van Woerden, H.: 1967, in H. van Woerden (ed.), 'Radio Astronomy and the Galactic System', IAU Symp. 31, 3.

Walker, M. F.: 1969, Astrophys. J. 155, 447. 